\title{
Reviewers 2020
}

Published online: 21 January 2021

(C) Springer Nature Switzerland AG 2021

Philosophy of Management thanks both the members of its distinguished editorial board and the following scholars for their excellent assistance to the journal by way of their service as reviewers in 2020:

Amir Abdulmuhsin

Robert Albin

Margaret Allen

Alexandre Bachkirov

Kit Barton

Enrico Beltramini

Caleb Bernacchio

Anindo Bhattacharjee

Kenneth Bohl

Siri Carson

Ruth Chadwick

Gareth Craze

Ananda Das Gupta

Joop de Zwart

Philipp Dorstewitz

Marian Eabrasu

Christopher Gohl

Agata Gurzawska

Graham Harman

Nathan Harter

Alicia Hennig

Matthias Huehn

Robert Hughes

Muatasim Ismaeel

Fahri Karakas
Feyyat Kaymaz

Gregory Latemore

Michael Loughlin

Yotam Lurie

David Lutz

Rob Macklin

Alan Malachowski

Mathias Naudin

Donald Nordberg

Ashly Pinnington

Daniele Ruggiu

Brian Rutherford

Martin Sand

Michael P. Schlaile

David Shaw

Robert Shaw

Stephen Sheard

Corey Steiner

Job Timmermans

Eva Tsahuridu

John Varlaro

David Vogel

David Wilson

Publisher's Note Springer Nature remains neutral with regard to jurisdictional claims in published maps and institutional affiliations. 